\title{
Bullous Systemic Lupus Erythematosus
}

National Cancer Institute

\section{Source}

National Cancer Institute. Bullous Systemic Lupus Erythematosus. NCI Thesaurus. Code C117104.

A manifestation of systemic lupus erythematosus with a widespread vesiculobullous eruption. 